\title{
Angular Distribution of Electron Temperature and Density in a Laser-Ablation Plume
}

\author{
B. Toftmann and J. Schou* \\ Department of Optics and Fluid Dynamics, Risф National Laboratory, DK-4000 Roskilde, Denmark \\ T. N. Hansen and J. G. Lunney \\ Department of Physics, Trinity College, Dublin 2, Ireland
}

(Received 22 April 1999)

\begin{abstract}
The angular distribution of electron temperature and density in a laser-ablation plume has been studied for the first time. The electron temperature ranges from 0.1 to $0.5 \mathrm{eV}$ and is only weakly dependent on the angle in the low-intensity range studied here. In contrast, the typical ion energy is about 2 orders of magnitude larger, and its angular distribution is more peaked about the target normal. The derived values of the electron density are in agreement with the measured values of ion density.
\end{abstract}

PACS numbers: 79.20.Ds, 52.70.-m, 81.15.Fg

Laser ablation of solids with nanosecond pulses of high intensity leads to complicated interactions of the laser beam with both the solid and the ablated material. Some of the fundamental physical features, such as the nature of the laser absorption in the vaporized material and acceleration mechanism for the ions, are not yet fully understood [1-4]. Nevertheless, the processing of solids by intense laser light and the production of thin films by pulsed laser deposition (PLD) are widely used techniques for a variety of materials $[5,6]$.

When a nanosecond laser pulse strikes a solid surface the rapid rise in temperature leads to intense evaporation of atoms and molecules from the solid. Even at relatively low intensities near the threshold for ablation $\left(0.2-1.0 \mathrm{GW} \mathrm{cm}^{-2}\right)$, it is observed that the ablated material is significantly ionized $[7,8]$, and the ions in the plasma plume have energies ranging up to several hundred $\mathrm{eV}$ [9]. At the end of the nanosecond laser pulse, the ablated material exists as a thin layer of plasma on the target surface. Initially the expansion of the plume is primarily driven by the plasma pressure gradients [2], but there may be an additional contribution from Coulomb repulsion between the ions if there is significant net loss of the more mobile electrons $[3,10]$. In any case, when the plume has propagated more than a few hundred $\mu \mathrm{m}$ from the target surface, the major part of the initial thermal energy in the plasma is converted to the directed kinetic energy of the ions, which are much more massive than the electrons $[11,12]$.

The energy distribution of the ions has been measured using time-of-flight (TOF) optical spectroscopy [13,14] and ion probes [4,7-9,15-17]. Mostly these ion probe measurements have been for the plasma flow close to the normal of the target surface. Recently, we measured the ion energy distribution for angles up to more than $60^{\circ}$, and it was observed that both the number and the average energy of the ions are strongly peaked about the target normal $[8,9]$. The electron component of the laser-induced plasma plume has been less widely studied, and to our knowledge only the electron temperature and density in the plasma flow perpendicular to the target surface have been reported
[17-20]. This is somewhat surprising, since in PLD the temperature and density of the electrons in the plasma will have a major influence on both the gas phase chemistry, between the target and the substrate, and the surface chemistry on the growing film.

For the first time we have measured the angular variation of the electron temperature and density in the plasma plume arising from the laser ablation of silver. The main outcome of our investigation was that the electron temperature varies more slowly as a function of angle than the ion energy distribution. Another important feature is that, in our intensity range, the electron temperature typically is between 0.1 and $0.5 \mathrm{eV}$, whereas the average ion flow energy is about 2 orders of magnitude larger.

The ablation was done using a frequency-tripled $\mathrm{Nd}$ :YAG laser $(355 \mathrm{~nm})$ directed at normal incidence onto a silver target in a vacuum of $10^{-8}$ Torr, in the same geometry as used earlier [8,9] (Fig. 1). The duration of the laser pulse was $6 \mathrm{~ns}$ and the fluence range $0.8-1.3 \mathrm{~J} \mathrm{~cm}^{-2}$, which corresponds to an intensity of $(1.3-2.2) \times 10^{8} \mathrm{~W} \mathrm{~cm}^{-2}$. The laser spot on target had an

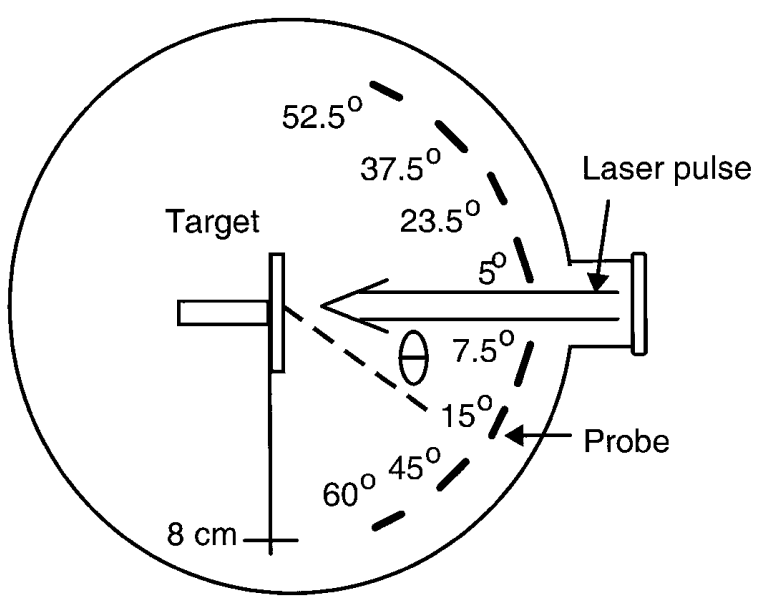

FIG. 1. The experimental setup showing the planar probes and the angle $\theta$ from the normal of a silver target. The laser wavelength is $355 \mathrm{~nm}$. 
area of $0.04 \mathrm{~cm}^{2}$, and was slightly elliptical $(b / a=1.6)$ with the minor axis, $a$, in the horizontal plane. The small ellipticity of the laser spot on target solely causes the ablation plume to be marginally wider in the horizontal rather than vertical plane [21]. The geometrical arrangement of the Langmuir probes used to measure the electron and ion signals is shown in Fig. 1, and is slightly modified compared with that described earlier [8,9]. Briefly, eight probes were positioned in a circular array in the horizontal plane around the laser spot at a distance of $8 \mathrm{~cm}$ and with angles varying from $5^{\circ}$ to $60^{\circ}$ with respect to the normal. The target-probe distance is slightly larger, but comparable to the target-substrate distance typically used in PLD. In contrast to our earlier work with cylindrical wire probes $[8,9]$, here we used planar probes, which are conceptually simpler and can be sensitive to the direction of the plasma flow $[18,22]$. Each probe was a $5 \times 5 \mathrm{~mm}^{2}$ square copper plate insulated on the rear side. All probes were oriented to face the irradiated spot on the target, i.e., perpendicular to the plasma flow.

The probes were biased with a variable voltage between -40 and $10 \mathrm{~V}$, and, while a measurement was made with one probe, all other probes, the silver target, and the vacuum chamber were grounded. The collected current was determined from the voltage signal developed across a $0.47 \Omega$ resistor. The bias was maintained using a $2 \mu \mathrm{F}$ capacitor, which was sufficient to avoid charge saturation of the system in the bias regime studied here. To avoid contamination of the target surface, five shots at $\sim 1 \mathrm{~Hz}$ were made for each measurement, but only the probe signal on the last shot was recorded [23]. Also the amplitude of the signal from a new target spot was found to fall to a constant amplitude after about 50 pulses. All of the measurements were taken after such target conditioning. The drift of the signals during the measurement of an individual probe for more than 200 pulses was typically less than $10 \%$ for the first $15 \mu \mathrm{s}$ and somewhat more for later times.

The electron temperature was determined on the basis of Langmuir probe theory [22] according to the analysis of Koopman and Segall $[18,19]$ which established the method of using Langmuir probes in the flowing plasma produced by laser ablation. Figure 2 shows the probe signals for $-2,-0.5,-0.1$, and $0.5 \mathrm{~V}$. At $-2 \mathrm{~V}$ the electrons are repelled completely from the probe, and the signal represents the time-of-flight spectrum of ions. (In fact, we used such signals in Refs. [8] and [9] to measure the ion energy spectra). Similar ion and electron signals are also reported in Ref. [24]. The signal obtained at $0.5 \mathrm{~V}$ consists largely of an electron signal superimposed on the signal due to ions with energies greater than $0.5 \mathrm{eV}$. The signal at $-0.1 \mathrm{~V}$, to which both electrons and ions contribute, is comparatively small, since the ion signal cancels much of the (negative) electron signal. In this region, where the electron and ion fluxes are comparable, the total electron flux can be deduced by adding the absolute ion saturation signal to the recorded signal. The ion saturation current

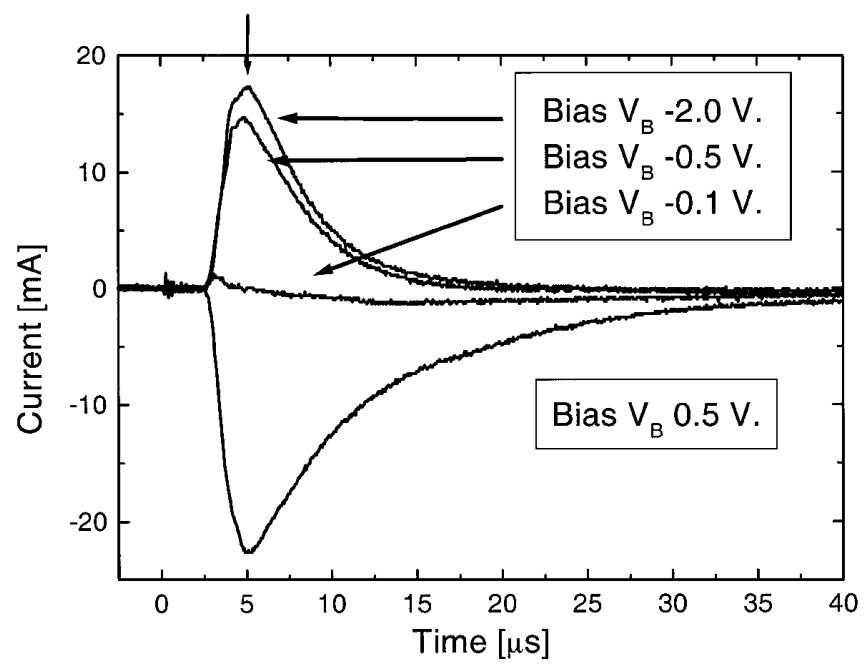

FIG. 2. Langmuir probe signals in the ion saturation region $(-2 \mathrm{~V})$ and in the transition regime $(-0.5,-0.1,0.5 \mathrm{~V})$ for the fluence $0.8 \mathrm{~J} \mathrm{~cm}^{-2}$ and the probe at $\theta=15^{\circ}$. The arrow indicates the time of maximum ion flux (used in Fig. 5).

was taken to be the average of the signals obtained in the bias region -40 to $-2 \mathrm{~V}$, where the values are nearly constant. The underlying assumption [18] is that the ion flow energy should be much higher than the probe potential and the Debye length much smaller than the probe dimension, as is the case here. Also the low particle density, of about $10^{11} \mathrm{~cm}^{-3}$, in the probe region (see below) means that any ions or neutrals backscattered or sputtered from the probes will not be collisionally coupled to the plasma, and thus the probes should not cause a significant perturbation of the plasma. The high electron current that may be drawn from the plasma for a large positive bias on a planar probe with a relatively large area imposes a practical upper limit for obtaining the full characteristic in the electron saturation region. The high electron currents have also prevented us from including higher laser intensities in the present study.

We have determined the electron temperature at different times after the front of the ablation plume has passed the probe; time is measured from when the laser strikes the target. For a homogeneous and isotropic plasma the electron saturation current for a positive bias is

$$
I_{e, \mathrm{sat}}=\frac{1}{4} e n_{e} \bar{v} A,
$$

where $n_{e}$ is the electron density, $\bar{v}$ is the average electron velocity, and $A$ is the probe area. In the transition region, as the probe voltage $V_{B}$ is decreased, an increasing number of electrons are repelled from the probe. For a Maxwellian energy distribution, $\bar{v}=\left(8 k T_{e} / \pi m_{e}\right)^{1 / 2}$, and the electron current is given by

$$
I_{e}=\exp \left[-e\left(V_{p}-V_{B}\right) / k T_{e}\right] I_{e, \text { sat }},
$$

where $V_{p}$ is the plasma potential, at which the probe has the same potential as the surrounding plasma. The transition regime can be identified as the linear part of the 


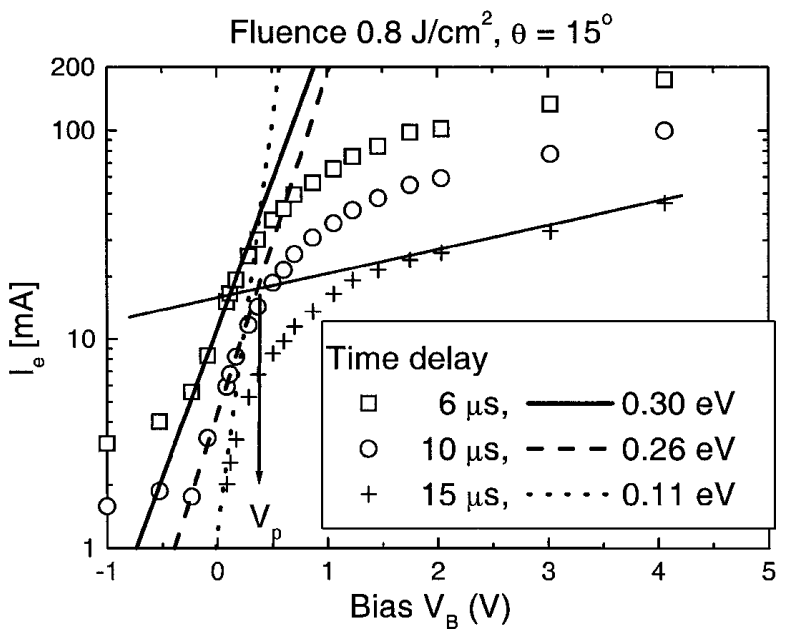

FIG. 3. The current-voltage characteristic for $0.8 \mathrm{~J} \mathrm{~cm}^{-2}$ and $\theta=15^{\circ}$ at the times 6,10 , and $15 \mu \mathrm{s}$. The electron temperature was determined from the slope in the transition region, according to Eq. (2). The electron saturation current $I_{e \text {, sat }}$ was taken as the value at the plasma potential, $V_{p}$, as shown for the $15 \mu$ s case.

semilogarithmic plot $\log I_{e}$ vs $V_{B}$. Examples of these characteristics for 6,10 , and $15 \mu$ s are shown in Fig. 3. In the negative bias region the net current is vanishing small since the ion contribution to the probe signal has already been subtracted. For most of the probes the instantaneous temperature increases up to a maximum, at about the time of the maximum of the ion signal (See Fig. 4), and thereafter decreases slowly with time.

The angular distributions of the $T_{e}$ at the time of maximum ion flux at $8 \mathrm{~cm}$ and at the time $15 \mu \mathrm{s}$ are shown in Fig. 5(b) for $0.8 \mathrm{~J} \mathrm{~cm}^{-2}$. $T_{e}$ does not vary much with angle and has a value about $0.2 \mathrm{eV}$. Figure 5(b) also shows the angular variation of the ion energy associated with the plasma flow at the time of maximum ion flux at the probes. This energy is about 2 orders of magnitude greater than the

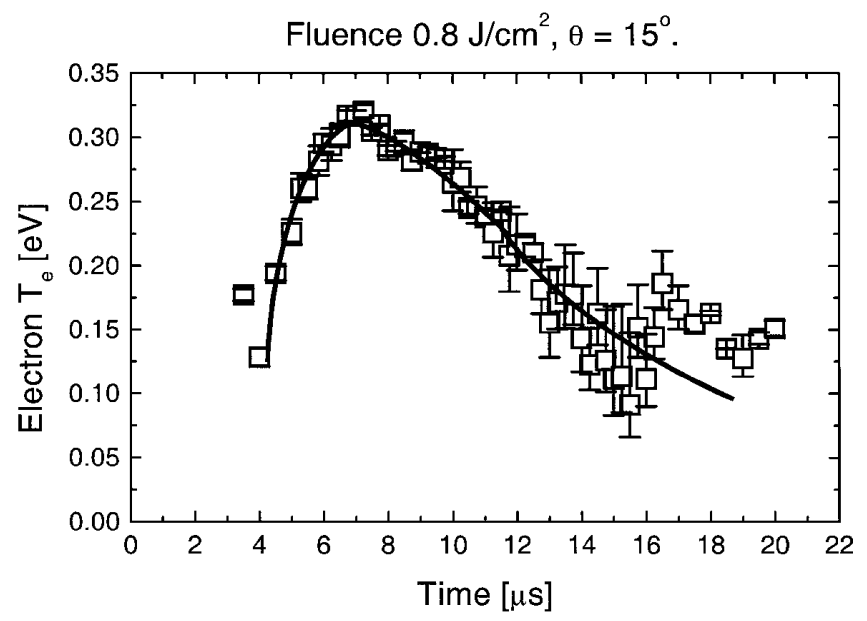

FIG. 4. Time variation of the electron temperature for the probe at $15^{\circ}$ and for $0.8 \mathrm{~J} \mathrm{~cm}^{-2}$. (The error bars indicate the accuracy of the fitting procedure in Fig. 3.) The solid line is a guide for the eye. electron temperature, and the variation with angle is much stronger than for the electron temperature.

Knowing $T_{e}$, and thereby the average electron velocity, allows us to determine the electron density by using Eq. (1) and the value of the electron saturation current $I_{e, \text { sat }}$. This is the value of the current when the probe is at the plasma potential $V_{p} . V_{p}$ is taken at the intersection of the linear fits to the electron saturation and transition regions of the $\log I_{e}-V_{B}$ characteristic [22]. Again, at the time of maximum ion flux, Fig. 5(a) shows the angle dependence of electron density at $8 \mathrm{~cm}$ for $0.8 \mathrm{~J} \mathrm{~cm}^{-2}$. Close to normal, it reaches a maximum value of about $4 \times 10^{11} \mathrm{~cm}^{-3}$, but falls sharply with increasing angle. At higher fluence the density increases, but the overall behavior is similar. Also plotted in Fig. 5(a) are the values of the ion density $n_{i}=I_{i} / e A v_{\mathrm{TOF}}$, at the time of maximum ion flux, as determined from the measured saturation ion current $I_{i}$ and the ion velocity $v_{\mathrm{TOF}}$. There is clearly very good agreement between the measured values of electron and ion density at practically all times and angles. Such agreement is to be expected for a quasineutral plasma, but also confirms the reliability of the measurements.

The temperatures measured here $(0.1-0.5 \mathrm{eV})$ are close to the values from Refs. $[25,26]$ which are obtained with
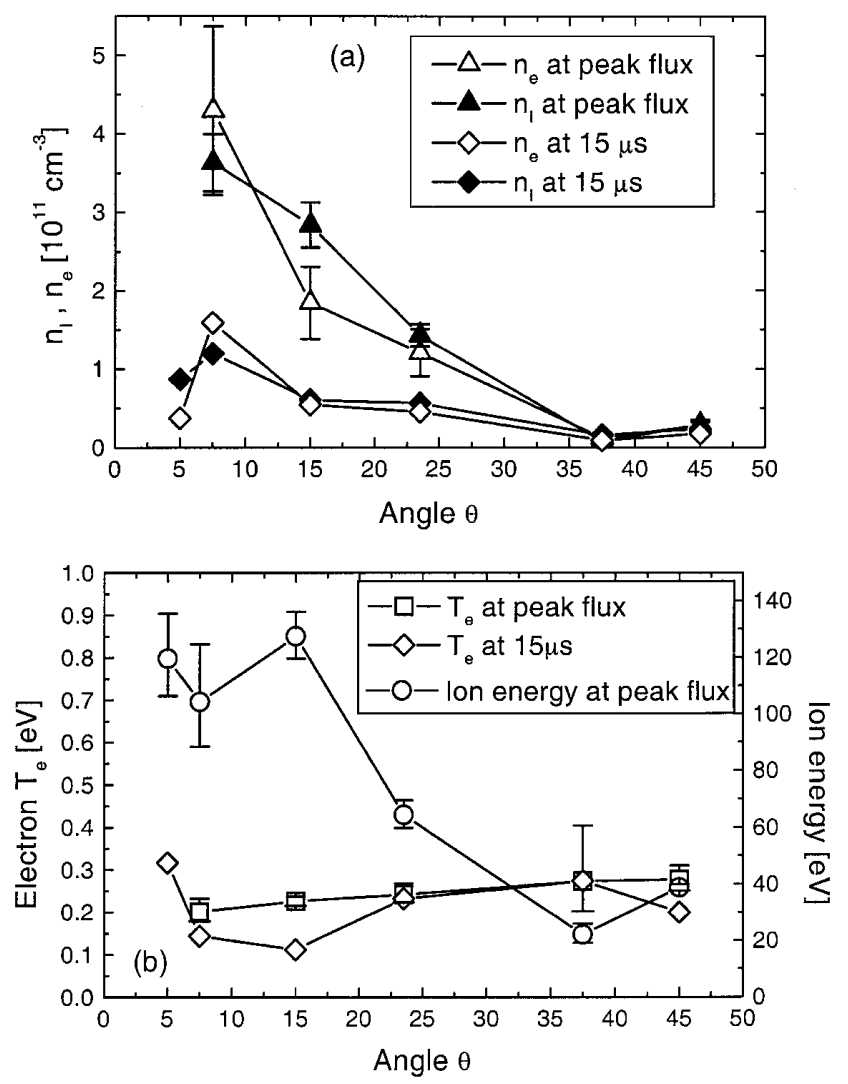

FIG. 5. The angular variation of (a) the electron and ion densities at the time of the ion peak position and at $15 \mu \mathrm{s}$ for $0.8 \mathrm{~J} \mathrm{~cm}^{-2}$; (b) the electron temperature $T_{e}$ for the same times as in (a) and the ion energy at the peak flux. (The signal from the probes at $52.5^{\circ}$ and $60^{\circ}$ was too small to be used.) 
a comparable laser fluence, but are considerably below those measured by Koopman [18] at much higher fluence $\left(900 \mathrm{~J} \mathrm{~cm}^{-2}\right)$. For the very late stages of the plasma expansion Hendron et al. [25] obtained good agreement between the values for $T_{e}$ as measured by a Langmuir probe and optical emission spectroscopy as well. The measured values of plasma density are broadly consistent with other reports in the literature [16,17], even though some of these are for higher fluence.

These measurements of plasma temperature and density provide us with a picture of the plume expansion and the energy balance for laser ablation at these low intensities. The plume of quasineutral plasma expands at high velocity away from the target such that the plasma velocity and ion energy are strongly peaked about the target normal. For the laser spot size and pulse duration used here, the angular variation of the plasma density and ion energy is consistent with the self-similar isentropic, adiabatic model of plume expansion developed by Anisimov et al. [21] and compared with experiment by Hansen et al. [27]. As the plume passes the probes, the maximum electron temperature is found at about the same time as the maximum ion signal is recorded. The measured value of the electron temperature at $8 \mathrm{~cm}$ is higher than the gas temperature in the model. However, this is not surprising since the electrons will collisionally decouple from the ions at a few $\mathrm{mm}$ from the target, and beyond that point are not cooled by the expansion. In any case, for all angles, the thermal electrons accompanying the ions carry only a small fraction of the energy in the plume.

In summary, we have used Langmuir probes to measure the angular distribution of the electron temperature and density in the ablation plume produced by laser irradiation of silver at up to $0.2 \mathrm{GW} \mathrm{cm}^{-2}$. Depending on the time after irradiation, the electron temperature lies in the range $0.1-0.5 \mathrm{eV}$, and is only weakly dependent on the angle of the plasma flow with respect to the target normal. In contrast, the average ion energy associated with the plasma flow is more sharply peaked about the target normal and is about 2 orders of magnitude higher. As is to be expected for a quasineutral plasma, there was very close agreement between the measured values of electron and ion densities. This experiment confirms that a Langmuir probe, which is a relatively simple diagnostic tool, can be used with confidence to measure the plasma parameters in the plume produced by pulsed laser ablation at low intensity.

The authors would like to thank J. J. Rasmussen and I. Weaver for helpful discussions. The work was supported by the Danish Natural Science Research Council, the European Union TMR (Contract No. ERBMRXCT980186), and the Enterprise Ireland International Collaboration Programme.

*Corresponding author.

Email address: j.schou@risoe.dk

[1] M. von Allmen and A. Blatter, Laser-Beam Interactions with Materials. Physical Principles and Applications (Springer, Berlin, 1995).

[2] T. P. Hughes, Plasmas and Laser Light (Adam Hilger Ltd., Bristol, UK, 1975).

[3] C. R. Phipps and R. W. Dreyfus, in Laser Ionization Mass Analysis, edited by A. Vertes, R. Gijbels, and F. Adams, Chemical Analysis Series Vol. 124 (Wiley, New York, 1993), pp. 369-431.

[4] J. G. Lunney and R. Jordan, Appl. Surf. Sci. 127-129, 941 (1998).

[5] D. H. Lowndes, D. B. Geohegan, A. A. Puretzky, D. P. Norton, and C. M. Rouleau, Science 273, 898 (1996).

[6] Pulsed Laser Deposition of Thin Films, edited by D. B. Chrisey and G. K. Hubler (Wiley, New York, 1994).

[7] P. E. Dyer, Appl. Phys. Lett. 55, 1630 (1989).

[8] T. N. Hansen, J. Schou, and J. G. Lunney, Europhys. Lett. 40, 441 (1997).

[9] T. N. Hansen, J. Schou, and J. G. Lunney, Appl. Phys. Lett. 72, 1829 (1998).

[10] R. W. Dreyfus, J. Appl. Phys. 69, 1721 (1991).

[11] A. F. Haught and D. H. Polk, Phys. Fluids 13, 2825 (1970).

[12] H. Puell, Z. Naturforsch. 25A, 1807 (1970).

[13] D. B. Geohegan and A.A. Puretzky, Appl. Surf. Sci. 96-98, 131 (1996).

[14] R. C. Issac et al., Appl. Surf. Sci. 125, 227 (1998).

[15] R. Jordan and J. G. Lunney, Appl. Surf. Sci. 127-129, 968 (1998).

[16] S. Amoroso et al., Appl. Surf. Sci. 106, 507 (1996).

[17] R. J. von Gutfeld and R. W. Dreyfus, Appl. Phys. Lett. 54, 1212 (1989).

[18] D. W. Koopman, Phys. Fluids 14, 1707 (1971).

[19] S. B. Segall and D. W. Koopman, Phys. Fluids 16, 1149 (1973).

[20] P. T. Rumsby and J. W. M. Paul, Plasma Phys. 16, 247 (1974).

[21] S. I. Anisimov, B.S. Luk'yanchuk, and A. Luches, Appl. Surf. Sci. 96-98, 24 (1996); S. I. Anisimov, D. Bäuerle, and B. S. Luk'yanchuk, Phys. Rev. B 48, 12076 (1993).

[22] N. Hershkowitz, in Plasma Diagnostics, edited by O. Auciello and D. L. Flamm (Academic Press, Boston, 1989), pp. 113-183.

[23] R. Dinger, K. Rohr, and H. Weber, J. Phys. D 17, 1707 (1984).

[24] S. Amoruso et al., Appl. Phys. Lett. 75, 7 (1999).

[25] J. M. Hendron et al., J. Appl. Phys. 81, 2131 (1997).

[26] I. Weaver et al., Rev. Sci. Instrum. 70, 1801 (1999).

[27] T. N. Hansen, B. Toftmann, J. Schou, and J. G. Lunney, Appl. Phys. A 69, S601 (1999). 